\title{
DYNAMICS OF DISTRIBUTION ASYMMETRY OF FIELD-ALIGNED CURRENTS DURING SUBSTORMS IN THE EQUINOX SEASON
}

\author{
V.V. Mishin \\ Institute of Solar-Terrestrial Physics SB RAS, \\ Irkutsk, Russia,vladm@iszf.irk.ru \\ V.M. Mishin ${ }^{\dagger}$ \\ Institute of Solar-Terrestrial Physics SB RAS, \\ Irkutsk, Russia
}

\author{
M.A. Kurikalova \\ Institute of Solar-Terrestrial Physics SB RAS, \\ Irkutsk, Russia, kurikalova@iszf.irk.ru
}

\begin{abstract}
On the basis of the magnetogram inversion technique, we study the dynamics of field-aligned current (FAC) distribution in the ionosphere of the Northern Hemisphere during the equinox, using data from the world magnetometer network. During selected substorms with an unchanged sign and module of the azimuthal IMF component, we have found a change of sign of the dawn-dusk asymmetry in the FAC intensity distribution. We attribute the change to the diurnal rotation of the geomagnetic dipole axis, the displacement of the terminator relative to the polar cap center, which caused the polar ionosphere illumination and conductivity to change. In addition, we have first detected a rapid change of the FAC asymmetry sign during the first substorm expansion phase when the terminator was near the polar cap center. We assume that such a fast dynamics
\end{abstract}

of the FAC asymmetry with the stable IMF azimuthal component during the equinox period might have been a consequence of the instability of the symmetry position of illumination and conductivity of two ionospheres due to diurnal rotation and strong interhemispheric asymmetry of the geomagnetic field, which might have caused the interhemispheric current flow.

Keywords: storm, substorm, field-aligned currents, dawn-dusk asymmetry.

\section{INTRODUCTION}

In the well-known model [Iijima, Potemra, 1978], obtained statistically from satellite data, field-aligned currents (FACs) fill two ring regions (low-latitude region 2 and high-latitude region 1), and near-polar region 0 , located inside the boundary of region 1 . According to the FAC sign, the system is partitioned into sub-regions chequerwise so that in region 1 FACs inflow on the dawn side and outflow on the dusk side, whereas in regions 2 and 0 surrounding it they have the opposite sign. The result of averaging over a large number of observations is that the system of FAC regions is symmetric relative to the noon-midnight meridian (see a more detailed FAC schematic with mesoscale irregularities in [Mishin et al., 2011]). As such, the ionospheric convection system usually consists of two vortices. During disturbances, the dusk vortex is more developed than the dawn one. This may be due, for example, to the near-terminator ionospheric conductivity gradient [Lyatsky, 1978; Atkinson, Hutchison, 1978; Lyatsky, Maltsev, 1983]. When geomagnetic activity increases, the entire convection system tends to rotate clockwise [Senior et al., 1990]. The FAC system also undergoes rotation whose direction during a substorm may change when passing from the growth phase to the expansion one [Mishin et al., 2019]. Several factors are responsible for the occurrence of the significant asymmetry in FAC and ionospheric convection distribution in both hemispheres and between them, as well as for the occurrence of interhemispheric field-aligned currents (IHCs). One of the causes for the asymmetry is related to the azimuthal component

$B_{y}$ of the interplanetary magnetic field (IMF). According to the open magnetosphere model [Lyatsky, 1978; Cowley, 1981; Lyatsky, Maltsev, 1983; Cowley, Lockwood, 1992], this IMF component when reconnected with the geomagnetic field causes an asymmetry in the distribution of the geomagnetic field, FAC, and convection in the ionosphere with respect to the noon-midnight meridian, as well as a displacement of the polar cap (PC) antiparallel to the IMF azimuthal component $\mathbf{B}_{y}$. The theoretical conclusions have been confirmed by observations (see references in the above studies, as well as in [Mishin, 1976, Mishin et al., 1992; Shirapov, Mishin 2009; Lukianova, Kozlovsky, 2013]. Superposing IMF $B_{y}$ on the geomagnetic field also leads to the formation of the asymmetric magnetic convection pattern in magnetotail lobes [Cowley, 1981; Tenfjord et al., 2015]. Optical satellite observations [Haaland et al., 2017; Østgaard et al., 2018] and early MIT results on the dynamics of electric fields and currents in two hemispheres [Lunyushkin et al., 2019] have shown that the auroral oval and PC in two hemispheres shift in opposite directions, depending on the sign of $B_{y}$. Both the FAC asymmetry and the IHC flow are attributed to the IMF $B_{y}$ effect. Thus, Stenbaek-Nielsen, Otto [1997] have assumed that IHCs in the auroral region produce the interhemispheric asymmetry in upward FACs. IHCs in this case result from the magnetic field shear (displacement, rotation) occurring in the magnetotail due to penetration of IMF $B_{y}$, and should primarily depend on its 
sign. Boroev, Gelberg [2001], Velichko et al. [2002a, b] have also observed the IMF $B_{y}$ effects: 1) asymmetry in the position of the longitude sector of substorm maximum intensity between the hemispheres; 2) a change in the ratio between bandwidths of downward and upward FACs as $B_{y}$ changes sign. The authors have suggested that in the region of break in the dawn-dusk current a tail of anomalous resistance, nonstationary field-aligned electric field and IHCs, whose direction depends on the sign of $B_{y}$, are formed.

Another possible cause for the asymmetry in the convection and FAC distributions is the influence of day-night ionospheric conductivity gradients [Lyatsky, 1978; Atkinson, Hutchison, 1978; Moses et al., 1987]. Lyatsky, Maltsev [1983] examined a model with a terminator crossing the PC center and showed that an abrupt change in the conductivity along the terminator line causes the dawn-dusk asymmetry in the ionospheric convection and FAC distributions. Adding the IMF component $B_{y}$ produces a displacement of convection flows in PC at the local time relative to noon. The combined effect of IMF $B_{y}$ and dipole tilt has been studied in [Reistad et al., 2019]. The authors have found out that the PC size is larger at opposite signs of the dipole tilt angle and IMF $B_{y}$ than in the case when their signs coincide. Suvorova [2017] has shown that the difference between coordinates of geomagnetic poles in Northern and Southern hemispheres induces a strong asymmetry in illumination and hence in wave conductivity of the polar ionosphere in summer and winter months. Theoretical works [Richmond, Roble, 1987; Benkevich et al., 2000; Benkevich, 2006; Lyatskaya et al., 2014, 2015] deal with quasi-stationary models of the electric circuit containing a generator and two paralleled ionospheres in the winter-summer season. The models predict that quasi-stationary IHCs can flow out of the regions with a steep conductivity gradient in the summer polar ionosphere, especially on the terminator. These IHCs can even shape a westward electrojet in the winter unlit ionosphere.

In the nighttime electric circuit model [Mishin et al, 2011; Mishin et al., 2016], the generator supplies the paralleled ionospheres of two hemispheres, which are additionally connected by region 2 field-aligned currents via partial ring currents, through region 1 nighttime cells. This connection at different conductivity in the two ionospheres should cause the FAC intensity dawn-dusk asymmetry in them, whose sign depends on the season.

Studying the dawn-dusk asymmetry is still an important avenue of research in magnetospheric physics [Forsyth et al., 2018; Liou, Mitchell, 2019]. The great progress in examining the ionospheric convection structure and dynamics is associated with the implementation of the SuperDARN project [Pettigrew et al., 2010]. The characteristic time scale of averaging from $\sim 10 \mathrm{~min}$ to 1 $\mathrm{hr}$, which is used in observational data processing, does not, however, give an instantaneous picture of ionospheric convection and FAC.

The magnetogram inversion technique (MIT) we apply can calculate two-dimensional distributions of electric fields and currents in the polar ionosphere every minute, providing insight into processes lasting a few minutes [Mishin, 1990]. For example, from data on sev- eral events the authors [Mishin et al., 2019; Mishin, Kurikalova, 2020] have revealed a phenomenon of rapid disintegration of the FAC system in the pre-midnight sector during the winter-type substorm expansion phase and associated it with the IHC flow between region 1 magnetically conjugate cells. In this paper, we delve into the dynamics of the FAC distribution asymmetry in the equinox season during the extended disturbed interval $02-16$ UT with a constant orientation of IMF $B_{y}$ having a relatively large value $\left(-6 \mathrm{nT}<B_{y}<-4 \mathrm{nT}\right)$. While there is no seasonal effect in the equinox, during the semidiurnal interval between the two substorms of interest the diurnal variation in the tilt angle of the geomagnetic dipole axis and hence in the illumination and ionospheric conductivity has time to manifest itself [Mishin, 1976]. The purpose of this paper is to figure out how the displacement of the terminator relative to the PC center, the change in the area of the sunlit PC region, the auroral oval, and ionospheric conductivity due to the diurnal rotation of Earth influence the dawn-dusk asymmetry in the FAC density distribution during the equinox. The study on time series of FAC density distribution maps, obtained by MIT with 1-min increment, has first demonstrated the possibility of changing the sign of the dawn-dusk asymmetry in the FAC intensity distribution for a few minutes during the substorm expansion phase. Besides MIT, maps of global FAC density distribution (with an option to calculate FAC intensities) can be obtained from AMPERE multisatellite program data [Coxon et al., 2018]. The procedure of statistical averaging of observations at a 10-min interval does not, however, allow us to trace the fast dynamics of FAC intensity during the expansion phase on a global scale. The same is true of the most advanced theoretical models of IHC [Lyatskaya et al., 2015] in the quasi-stationary approximation.

\section{GEOMAGNETIC CONDITIONS ON APRIL 6, 2000}

The April 6, 2000 event has been studied in several papers [Mishin et al., 2010; Mishin et al., 2011, Mishin, Kurikalova, 2020]. In this paper, we examine the semidiurnal interval before the sudden commencement (SSC) of a strong magnetospheric storm recorded at 16:40 UT. Figure 1 shows variations in solar wind parameters and variable part of the open magnetic flux through PC $\Psi_{1}=\Psi-\Psi_{0}$. Here, $\Psi_{0}$ is the magnetic flux value before a substorm. The total magnetic flux $\Psi$ through $\mathrm{PC}$ is $\Psi=\int B(r) d s$, where $B(r)$ is the dipole geomagnetic field at a height of $115 \mathrm{~km}$, and $S$ is the polar cap area. During the interval of interest, two substorm activations occurred, with onsets of the growth phase at $\sim$ 02:00 and $\sim 12: 00$ UT. During these activations, the solar wind dynamic pressure varied insignificantly $\left(P_{\mathrm{d}} \sim 1-2 \mathrm{nPa}\right)$, except for a short burst around 11:30 UT. In the interval of the first substorm, $\sim 02: 00$ UT, the southward and azimuthal IMF components enhanced. This caused the onset of the substorm growth phase. The subsequent sufficiently large negative values of the IMF components $B_{z}=B_{y} \sim-6 \mathrm{nT}$ were accompanied by a rapid transfer of the magnetic flux to magnetotail lobes and hence by an increase in the magnetic flux $\Psi$ 
6 April 2000
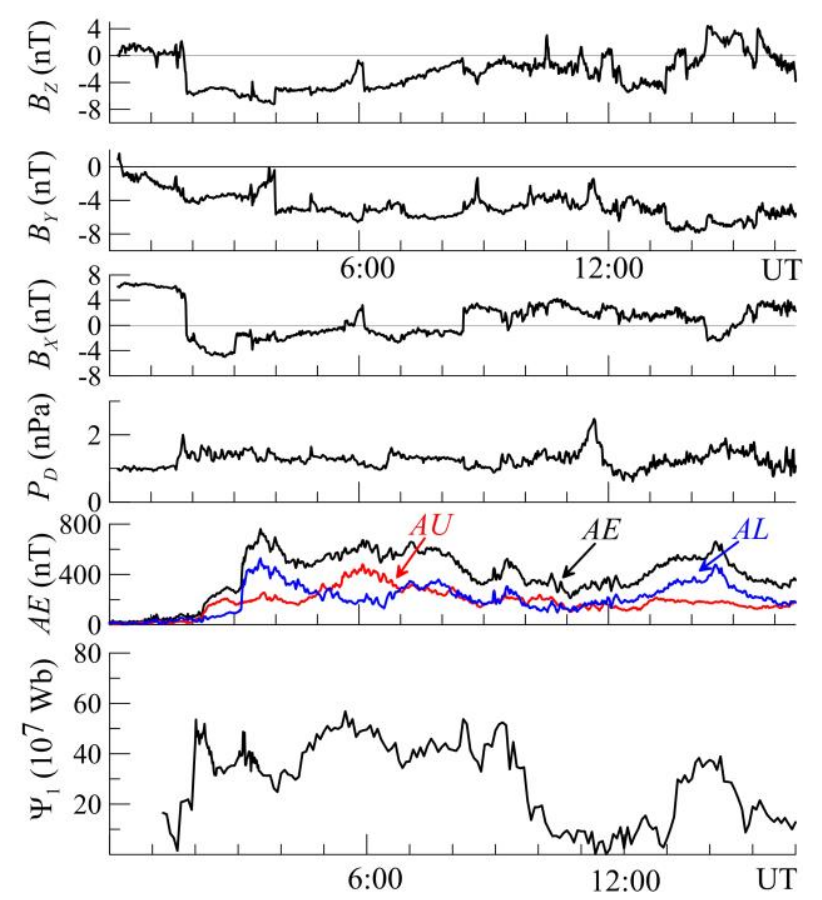

Figure 1. Variations in IMF components, solar wind dynamic pressure $P_{\mathrm{d}}, A E$ index, variable part of the polar cap magnetic flux $\Psi_{1}$ before SSC on April 6, 2000

through PC. At 03:10 UT, the expansion phase began a result of the reconnection and subsequent dipolarization of the magnetic field in the tail, which appeared as a sharp drop in the PC magnetic flux $\Psi$ and as an enhancement of the auroral activity indices $A L, A E$.

Throughout the interval of the second substorm activation (12-16 UT), IMF $B_{y}$ had steadily negative values near $B_{y}=-6 \mathrm{nT}$. The slow growth in $A E$ was followed by a more rapid increase in $\Psi$, which began around 13:00 UT. The IMF component $B_{z}$ sharply turned northward at $\sim 13: 20$ UT and became positive after 14:00 UT. In the interval 12:30-14:35 UT, the $A E$ index increased from 300 to $660 \mathrm{nT}$, followed by an increase in the magnetic flux $\Psi$ at 13:00-14:15 UT. At 14:15 UT, $\Psi$ began to decrease and $A L, A E$ began to increase, i.e. the substorm expansion phase commenced.

\section{METHOD}

We apply the original version of the magnetogram inversion technique (ISTP MIT), developed in the 1970s [Mishin, 1990]. MIT uses 1-min geomagnetic data from the global network of ground-based stations and can calculate not only two-dimensional maps of electric potential distribution, integral conductivities, horizontal and field-aligned currents, but is also capable of identifying and studying inhomogeneous structures mesoscale cells. MIT also provides a way to explore the dynamics of FAC intensities in each region and the dynamics of the PC magnetic flux [Mishin et al., 2011].

We use MIT for calculating time series of FAC density distribution maps with a 1-min increment. From these maps we find boundaries of large-scale FAC regions R0, R1, R2 (thick black lines in Figure 2),

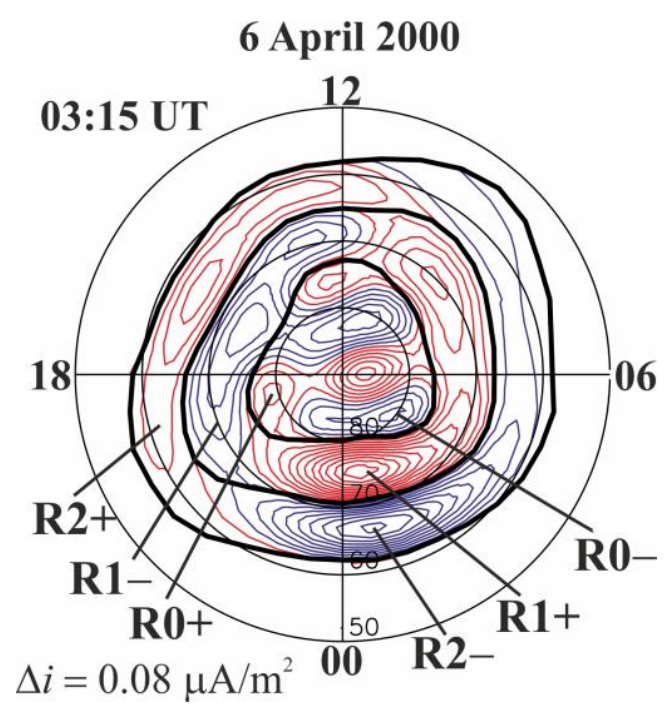

Figure 2. A map of two-dimensional distribution (geomagnetic latitude - geomagnetic local time) of the FAC density $i_{N}$ in the ionosphere of the Northern Hemisphere. Thick black lines indicate boundaries of FAC regions $\mathrm{R} 0+(-), \mathrm{R} 1+(-$ ), R2+(-). Signs "+", "-" correspond to downward/upward FACs

values of net currents - FAC intensities of both signs in each region, as well as the PC area $S$ and the magnetic flux $\Psi$ transferred though it.

Figure 2 gives an example of FAC density distribution map. In region 1, FAC inflows (outflows) in the dawn (dusk) sector; in regions 2 and 0 , vice versa.

\section{DAWN-DUSK ASYMMETRY IN FAC INTENSITY DISTRIBUTION}

Using FAC density distribution maps, we plotted (Figure 3 ) variations in the intensity of downward and upward FACs in regions 1 and 2 according to the classification [Iijima, Potemra, 1978] during two substorm activations at 02-16 UT on April 6, 2000.

The top panel shows that at 03:35 UT there are simultaneously maximum $A E$ and intensities of downward FAC in region $1\left(I_{\mathrm{R} 1+}\right)$ and upward FAC in region $2\left(I_{\mathrm{R} 2-}\right.$ ) in the dawn sector. At the same time, intensities of region 1 upward FACs and region 2 downward FACs in the dusk sector are several times lower than in the dawn sector. So, in the interval 03:10-04:00 UT during the expansion phase, the FAC asymmetry $I_{\text {dawn }}>I_{\text {dusk }}$ takes place. FAC distribution of this type [Mishin et al., 2015b] was named winter-type distribution.

The bottom panel for the maximum $A E$ index at 13:30 UT exhibits maximum FAC intensities $I_{\mathrm{R} 1-}$ and $I_{\mathrm{R} 2+}$ in regions $\mathrm{R} 1$ and $\mathrm{R} 2$ in the dusk sector. In the dawn sector, meanwhile, $I_{\mathrm{R} 1+}, I_{\mathrm{R} 2-}$ are lower. Thus, in the interval 10:00-16:00 UT there is the FAC asymmetry $I_{\text {dusk }}>I_{\text {dawn }}$. This FAC distribution corresponds to the summer-type FAC distribution in the Northern Hemisphere [Mishin et al., 2015a].

The upper panel to the left also indicates that the intensity of the region 1 downward FAC $I_{\mathrm{R} 1+}$ is first lower than that of the upward FAC $I_{\mathrm{R} 1-}$ (to around $03 \mathrm{UT}$ ) $\left(I_{\mathrm{R} 1+}<I_{\mathrm{R} 1-}\right)$, and then, in the interval 03:00-04:00 UT it, 
6 April 2000
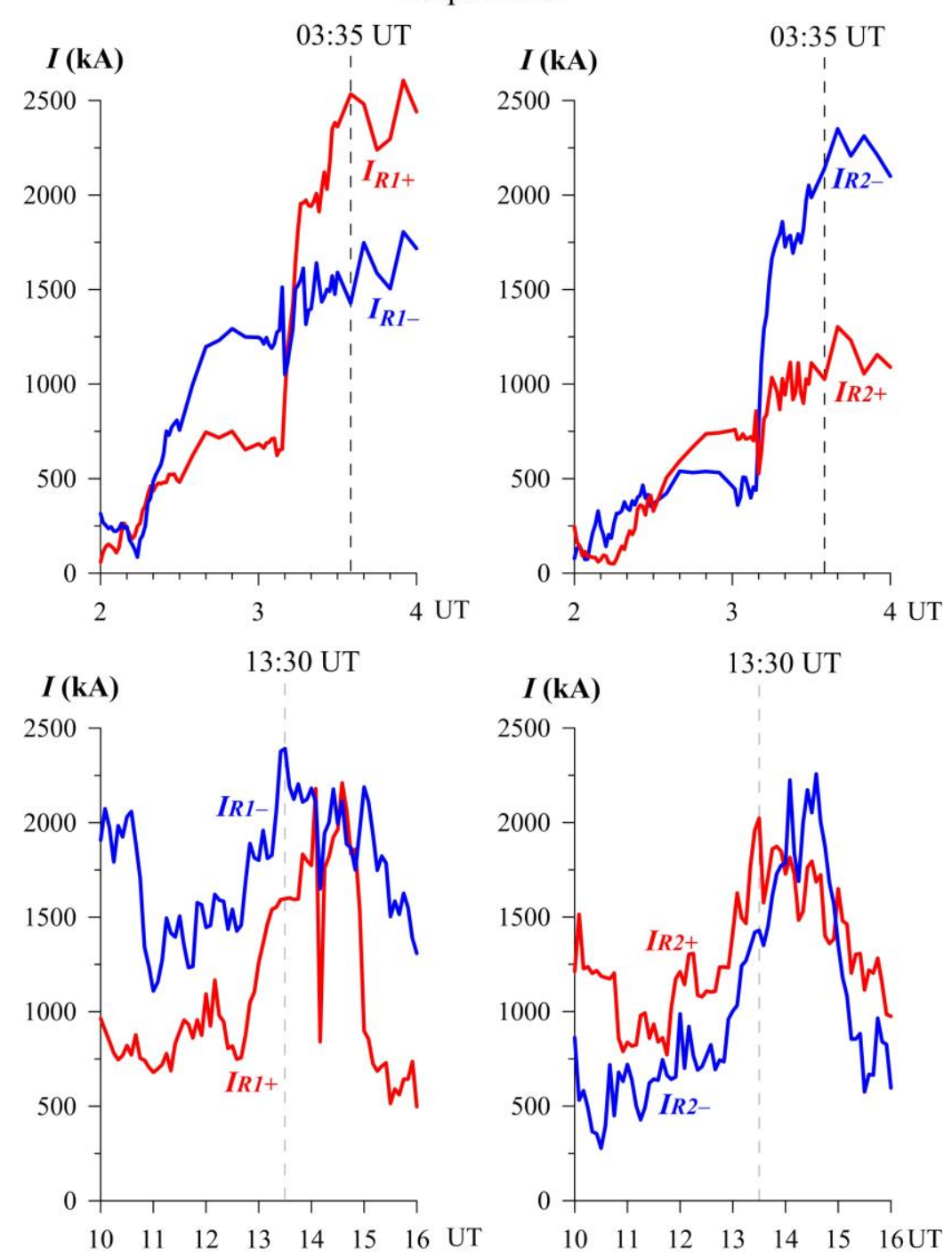

Figure 3. FAC intensity variations in region 1 (left) and in region 2 (right) in the intervals 02-04 UT (top) and 10-16 UT (bottom)

on the contrary, is much higher than that of the region 1 upward FAC $I_{\mathrm{R} 1+}>I_{\mathrm{R} 1-}$. Hence, there is a transition from the summer-type FAC distribution (in the interval 02:00-03:00 UT) to the winter-type one (in the interval 03:00-04:00 UT).

The bottom panel to the left shows that the intensity of region 1 upward FAC exceeds that of downward FAC almost throughout 12:00-16:00 UT, i.e. the summer-type FAC distribution in this interval persists. The only exception is a small interval around the FAC maximum when intensities of downward and upward fieldaligned currents are equal. A similar symmetrization of configuration with increasing magnetospheric activity is shown in [Reistad et al., 2018].

Let us emphasize once again that at 02-16 UT, i.e. during both substorms, azimuthal IMF remained almost unchanged, but the sign of the prevailing dawn-dusk asymmetry in the FAC intensity distribution changed during the transition from the first substorm to the second one: from the winter type $\left(I_{\mathrm{dawn}}>I_{\mathrm{dusk}}\right)$ in the interval 02-04 UT to the summer type $\left(I_{\text {dusk }}>I_{\text {dawn }}\right)$ in the interval 12-16 UT.

\section{TERMINATOR DYNAMICS}

The plane of the Earth equator is known to make an angle of $23^{\circ} 27^{\prime}$ with the orbit plane. As a result, PC is sunlit in summer. The opposite situation occurs in winter. During the equinox, half of the Earth surface is sunlit, lengths of day and night are nearly equal. Motion of the terminator in geomagnetic coordinates and PC illumination are determined by diurnal oscillation of the geomagnetic dipole axis relative to the rotation axis. We assume that the FAC distribution asymmetry may be related to the change in PC and auroral oval illumination in the events of April 6, which was caused by the diurnal rotation of Earth.

Let us trace the position of the terminator on April 6, 2000 . The tilt angle of the geomagnetic dipole axis $\Phi_{\text {tilt }}$, which determines the position of the terminator, on April 6 varied within $-4.72^{\circ} \leq \Phi$ tilt $\leq 17.68^{\circ}$. It took the smallest value $\Phi_{\text {tilt }}=-4.72^{\circ}$ at $04: 43$ UT, the largest value $\Phi_{\text {tilt }}=17.68^{\circ}$ at $16: 43$ UT. The angle $\Phi_{\text {tilt }}$ was approximately equal to zero at 01:05 and 08:22 UT. The tilt angle of the geomagnetic dipole axis was calculated 
from the following formula [e.g. Shue, 1993]:

$$
\begin{aligned}
& \Phi_{\text {year }}=23.4 \cos [(\mathrm{DAY}-172) 2 \pi / 365.25] ; \\
& \Phi_{\text {day }}=11.2 \cos [(\mathrm{UT}-16.72) 2 \pi / 24] ; \\
& \Phi_{\text {tilt }}=\Phi_{\text {year }}+\Phi_{\text {day }} .
\end{aligned}
$$

Here, the tilt angle of the geomagnetic dipole axis is the sum of the annual $\left(\Phi_{\text {year }}\right)$ and daily $\left(\Phi_{\text {day }}\right)$ tilt angles of the geomagnetic dipole axis; DAY is the number of day in the year. Between 01:05 and 08:22 UT, $\Phi_{\text {tilt }}<0$ (the terrestrial dipole axis is tilted from the Sun), i.e. it is the interval of a lower illumination of the Northern Hemisphere (winter). Between 08:02 and 01:05 UT, $\Phi_{\text {tilt }}>0$ (the terrestrial dipole axis is tilted to the Sun), i.e. it is the interval of a higher illumination (summer).

The former interval 02-04 UT occurred when the PC illumination was lower. In Section 3, we observed a change in the asymmetry sign at the onset of the first substorm expansion phase (03:10 UT). During this substorm, the dawn-dusk asymmetry changed sign when the terminator passed almost through the geomagnetic pole (Figure 4).

While IMF $B_{y}$ took negative values from $00 \mathrm{UT}$ and its mean value was $\left\langle B_{y}\right\rangle \sim-3 \mathrm{nT}$ between 02 and $04 \mathrm{UT}$, at 02:50 UT the displacement of the oval and PC relative to the noon-midnight meridian was not observed yet, whereas at 03:16 UT it could be seen already. This characterizes the time of PC response to the IMF $B_{y}$ effect.

The latter interval 12-16 UT happened when PC was more sunlit, the terminator was to the south of the geomagnetic pole, and the asymmetry $I_{\text {dawn }}<I_{\text {dusk }}$ prevailed. In this interval, the average modulus of the azimuthal component doubled $\left\langle B_{y}\right\rangle=-6 \mathrm{nT}$, with a significant displacement of the oval (regions 1 and 2) and PC from dawn to dusk (against the direction of $B_{y}$ ) relative to the noon-midnight meridian (Figure 5).

Figure 6 shows the effect of IMF $B_{y}<0$ on the duskward displacement of $\mathrm{PC}$, which is more pronounced for the second substorm. Comparison with Figure 5 suggests that the dawn-dusk shift of the dusk PC boundary becomes smaller. Thus, during the substorm expansion phase there is a tendency for the dawndusk asymmetry to be minimized with respect to the noon-midnight meridian.

Figure 7 depicts variations in the ratio between areas of sunlit and unlit PC regions $S_{\text {light }} / S_{\text {dark. }}$. You can see (left) that in the interval of the first substorm (02-04 UT) the areas of the sunlit and unlit PC regions are approximately equal $\left(S_{\text {light }} / S_{\mathrm{dark}} \sim 1\right)$. In the interval of the second substorm (12-15 UT), the area of the sunlit PC region considerably exceeds that of the unlit region $\left(S_{\text {light }} / S_{\text {dark }}>>1\right)$. A minimum ratio between $S_{\text {light }} / S_{\text {dark }}=2$ is observed near the peak of the second substorm expansion phase (hereinafter: the substorm peak is the maximum FAC intensity and $A E$ ).

The dipole tilt angle at the peak of the expansion phase of the first (03:35 UT) and second (13:30 UT) substorms took the following values:

$\Phi_{\text {tilt }}(03: 35$ UT $)=-4.22^{\circ}$;

$\Phi_{\text {tilt }}(13: 30$ UT $)=13.93^{\circ}$;

The difference between the dipole tilt angles at 03:35 and 13:30 UT is:
$\Delta \Phi_{\text {tilt }}(03: 35 \div 13: 30 \mathrm{UT})=18.15^{\circ} .^{\prime}$

Note an interesting fact: a change in the dipole tilt angle and the terminator position between the two substorm activations is only $\sim 18^{\circ}$. The ratio between areas of the sunlit and unlit PC regions, however, differs considerably due to the fast dynamics of PC during the second substorm.

\section{DISCUSSION}

We have analyzed two substorm intervals before the April 6, 2000 magnetospheric storm. For each of them, variations in the intensity of downward and upward FACs have been plotted for dusk and dawn sectors of each of the three FAC regions. We have shown that during the equinox season there are FAC distributions of two types we called summer-type FAC distribution and winter-type FAC distribution [Mishin et al., 2015b]. Recall that in the 02-16 UT interval of the April 6, 2000 event IMF $B_{y}<0$ does not change sign. There are, nonetheless, two types of FAC intensity distribution in the dawn and dusk sectors of the FAC regions $\left(I_{\text {dawn }} / I_{\text {dusk }}<1\right.$ and $I_{\text {dawn }} / I_{\text {dusk }}>1$ ). Thus, the change of the FAC distribution type occurs at constant IMF $B_{y}$ sign.

We have examined the dynamics of the tilt angle of the geomagnetic dipole axis, the relationship of the displacement of the terminator relative to the pole and the degree of PC illumination with FAC distribution. We have shown that when the dipole tilt angle is positive and the area of the sunlit PC region is more than twice the area of its unlit region in R1, R2 the summer-type asymmetry $I_{\text {dusk }}>I_{\text {dawn }}$ occurs (Figure 3 (10-16 UT), Figure 7, right; Figures 5 and 6, right).

During the first substorm activation, the areas of the sunlit and unlit PC regions are nearly equal, both types of the FAC distribution asymmetry are equally probable (Figure 3, top and Figure 7, left), and during the expansion phase the type of asymmetry can be rapidly changed. We managed to obtain the fast FAC distribution dynamics due to the global network of groundbased magnetometers with 1-min recording speed used in MIT. Since, as we noted in Introduction, the time resolution of the most expensive satellite project AMPERE is $10 \mathrm{~min}$, from its data it is difficult to watch the short-term equalizing of the asymmetry we described and much less the rapid change of its sign during the expansion phase.

In Introduction, we have described the main factors of the occurrence of the dawn-dusk asymmetry in FAC distribution: the effect of the azimuthal IMF component, ionospheric conductivity irregularities and their attendant IHCs. In the event we considered, IMF $B_{y}$ remained unchanged during both substorms, and the change of sign of the dawn-dusk asymmetry in FAC distribution occurred only in the first substorm with nearly equal areas of sunlit and unlit PC regions $S_{\text {light }} / S_{\text {dark }} \sim 1$. We therefore assume that in this case one of the possible causes for the rapid change of the FAC distribution type is transient IHCs. The possibility of the formation of IHCs under equinox conditions due to diurnal variation in the terminator position relative to geomagnetic poles and the occurrence of inequality between 
6 April 2000
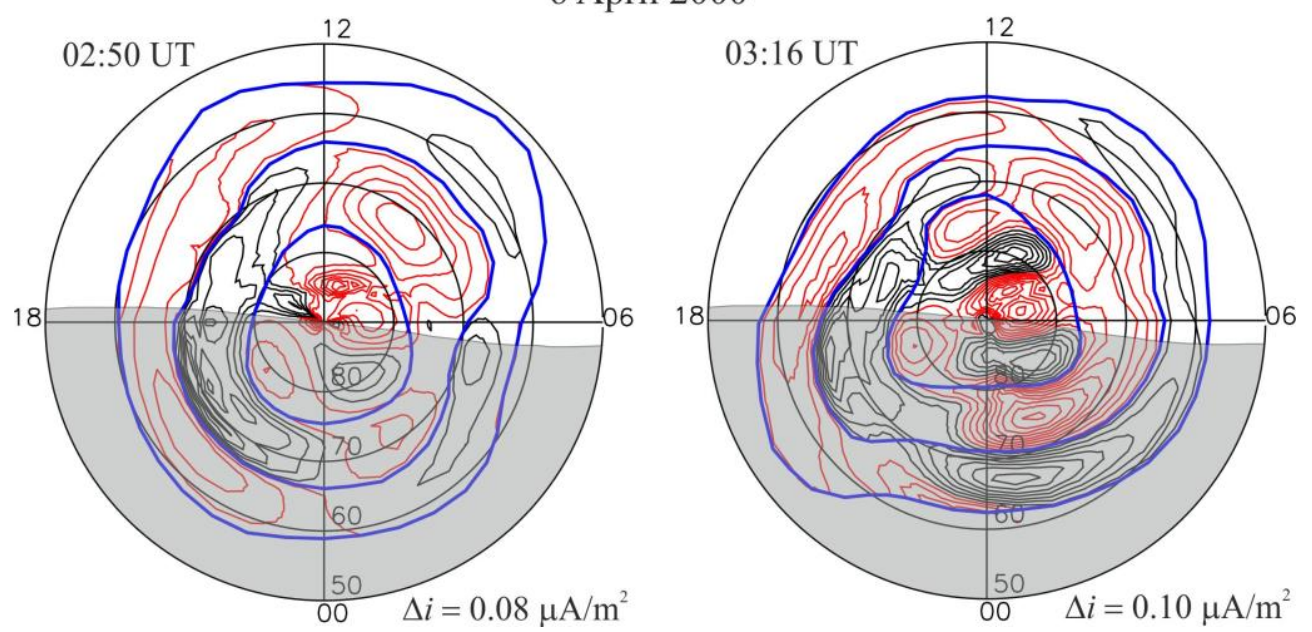

Figure 4. Examples of maps of FAC density distribution, position of the terminator and FAC region boundaries in the late growth phase (left) and early active phase of the first substorm (02-04 UT). The PC dawn-dusk shift (against the direction of IMF $B_{y}$ ) managed to become stable at the second moment

\section{April 2000}
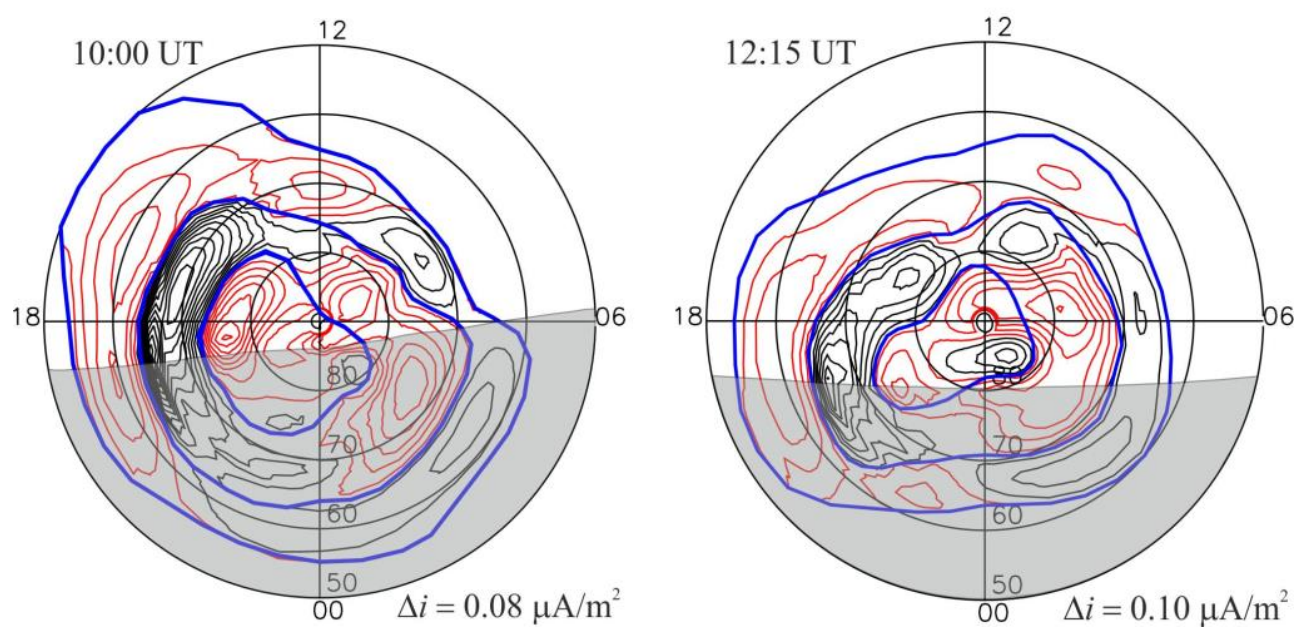

Figure 5. The same as in Figure 4 before and during the growth phase of the second substorm activation at 12-16 UT

6 April 2000
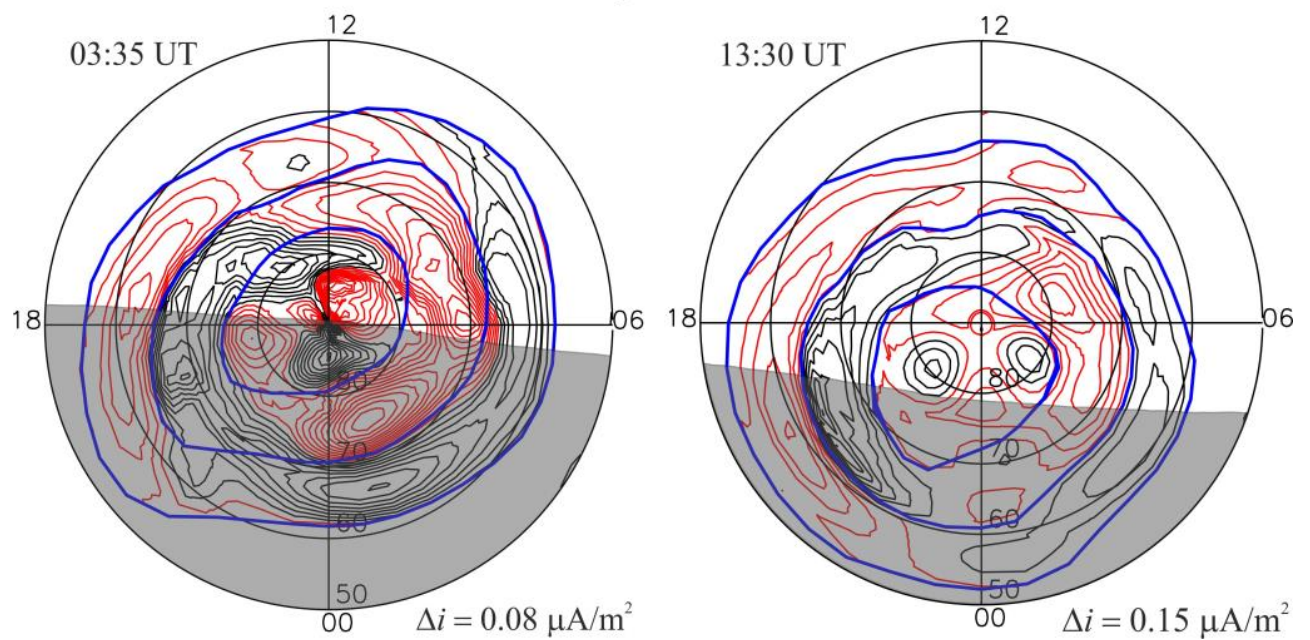

Figure 6. Position of the terminator on a FAC density distribution map at the peak intensity of region 1 FAC during the expansion phase of the two substorms. Moments 03:35 UT (left) in the first interval 02-04 UT (approximately equal light and dark PC regions $\left.\left(S_{\text {light }}=S_{\text {dark }}\right)\right)$ and 13:30 UT in the second interval $12-16$ UT $\left(S_{\text {light }} / S_{\text {dark }}=2\right)$ 


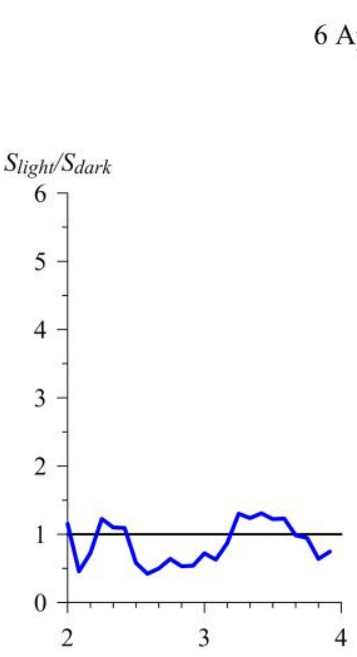

6 April 2000

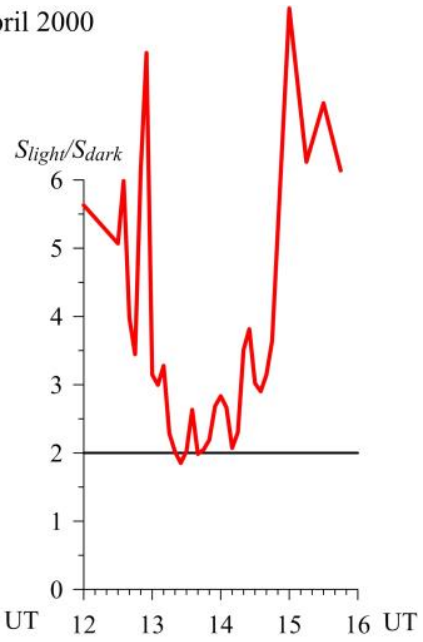

Figure 7. Variations in the ratio between areas of sunlit and unlit PC regions $S_{\text {light }} / S_{\text {dark }}$ during two substorms

conductivities in two hemispheres has been discussed by Benkevich et al. [2000]. We will show qualitatively, by analogy with [Benkevich et al., 2000; Mishin et al., 2019], that even during the equinox the symmetry between illumination and conductivity of the ionosphere is unstable, which may cause IHCs.

In the global electric circuit containing a generator in the magnetosphere and two loads (ionospheres), the generator through region 1 supplies via a FAC pair the paralleled ionospheres of two hemispheres in the morning, with currents in the ionosphere flowing along the parallel, flowing out of them in the evening, and closed on the generator (see Figure 7 in [Mishin et al., 2011]. Suppose that during the equinox the terminator is located at the center of both PCs, $S_{\text {light }} / S_{\text {dark }}=1$, and ionospheric conductivities of two hemispheres coincide, therefore equal currents flow through them. When the terminator diverges from the PC center (pole), the symmetry is disturbed - the equality between effective resistances (conductivities) is violated. At the intersection with R1, the terminator causes a longitudinal conductivity gradient in it, thereby, in accordance with Ohm and Kirchoff laws, causing IHC to flow from the Southern Hemisphere with higher conductivity to the Northern Hemisphere with lower conductivity through the geomagnetic field line with infinite conductivity. In the absence of such a longitudinal conductivity gradient along the parallel inside region 1 , even if there is a strong inequality in conductivities between hemispheres, current (i.e. IHC) cannot occur between them (see details in Figure 3.2 in [Benkevich, 2006]). IHC is a current ensuring a rapid transition to a new distribution of conductivity and ionospheric currents. If there is no desired abrupt change in conductivity along the parallel, IHC does not arise. Let us estimate now the order of IHC magnitude. Imagine a magnetic flux tube in the dusk sector, which connects two magnetically conjugate regions of FAC region 1 in the vicinity of the intersection of the terminator with the region 1 pole boundary in both ionospheres. Similarly to [Stenbaek-Nielsen, Otto, 1997; Mishin et al., 2019]), superimpose IHC flowing from the summer ionosphere to the winter one on this tube. Such IHC may produce additional downward FAC in the winter hemisphere, thereby reducing the observable total upward FAC in this region, which is associated with a weakening of $I_{\mathrm{R} 1-}$ (see Figure 3 ) in the winter interval 03:05-04:00 UT. Conversely, IHC in the summer hemisphere may induce additional downward FAC, thus increasing the observable total upward FAC (an increase in $I_{\mathrm{R} 1-}$ (see Figure 3 ) in the summer interval 02:15-03:05 UT).

Assuming according to the data from [Lunyushkin et al., 2019] that $I_{\text {summer }} \simeq 2 I_{\text {winter }}$, we can estimate the intensity $I_{\mathrm{IHC}} \cong 1 / 3 I_{\mathrm{R} 1-}(\sim 400 \mathrm{kA}$ at the peak of the substorm).

Since FACs transfer Alfvén waves [Lyatsky, Maltsev, 1983; Kostarev et al., 2021] with a characteristic velocity in the magnetosphere $V_{\mathrm{A}}=1000 \mathrm{~km} / \mathrm{s}$, the time of IHC flow from one PC to another in the vicinity of the 06-18 UT meridian $t \sim 3-5 \mathrm{~min}$, which is comparable to the time of the rapid change in $I_{\text {dusk }} / I_{\text {dawn }}$ (see Figure 3 around 03:10 UT). If we do not assume that IHCs flow, it takes FACs in two hemispheres enormously more time to change in the global circuit through the magnetotail. It should be noted that $S_{\text {light }} / S_{\text {dark }}$ varies within 1 on the interval 02-04 UT (Figure 7), and besides the rotation of the geomagnetic dipole axis an additional factor of the symmetry loss is the strong interhemispheric asymmetry in the geomagnetic field, which causes the conductivity asymmetry [Suvorova, 2017]. The transition from one FAC asymmetry type to another with occurrence of IHC might have therefore occurred at any point of the interval of the first activation expansion phase. Moreover, it is difficult to expect an exact implementation of the proposed mechanism from data obtained in one hemisphere, although the idea about the role of transient IHCs in the rapid change of FAC asymmetry sign under equinox conditions seems very plausible.

Now let us turn to our findings about the IMF $B_{y}$ effect on the position of PC (see Figures 4-6), which are consistent with the known fact that PC shifts in the Northern Hemisphere against the direction of IMF $B_{y}$ (to dusk), as predicted by the model [Cowley, Lockwood, 1992] and observations [Mishin et al., 1992; Lukianova, Kozlovsky, 2013].

Note that during the expansion phase regardless of change of FAC distribution asymmetry sign it disappears for a short time. The tendency for the PC dawndusk asymmetry relative to the noon-midnight meridian to minimize during the substorm expansion phase is well-known, has been used in [Mishin et al., 2011], and is also consistent with recent data [Østgaard et al., 2018; Lunyushkin et al., 2019].

\section{CONCLUSION}

Our main results are as follows:

1. In regions 1 and 2, the dawn-dusk asymmetry in FAC intensity distribution changes sing between the two intervals on April 6, 2000 for a few minutes during the first substorm expansion phase (at $S_{\text {light }} \sim S_{\text {dark }}$ ).

2. Since IMF $B_{y}$ on April 6, 2000 (02-16 UT) remained practically unchanged, a possible cause for the reversal of 
the direction of the dawn-dusk FAC asymmetry is the swing of the geomagnetic dipole axis relative to the Earth rotation axis, which alters the position of the terminator and the degree of PC and auroral oval illumination.

3. During the substorm expansion phase regardless of the change of FAC distribution asymmetry sign, it disappears for a short time.

4. The effect of the IMF negative azimuthal component shows up in the dawn-dusk displacement of PC (consistent with predictions of the open magnetosphere model [Cowley, Lockwood, 1992]) in more than two hours after its occurrence at 0 UT. This displacement slightly decreases during the second substorm expansion phase on April 6, 2000 (12-16 UT).

5. When the area of the sunlit PC region $S_{\text {light }}>S_{\text {dark }}$ is larger, the summer-type FAC distribution asymmetry $\left(I_{\text {dusk }}>I_{\text {dawn }}\right)$ predominates.

6. We assume that in the interval 02-04 UT at equal illumination of PC $\left(S_{\text {light }} \sim S_{\text {dark }}\right)$ the rapid change in the FAC distribution dawn-dusk asymmetry may be associated with interhemispheric current flow. The IHC intensity can be estimated as $I_{\mathrm{IHC}} \sim 1 / 3 I_{\mathrm{R} 1-}(\sim 400 \mathrm{kA}$ at the peak of the substorm).

In conclusion, note that the results about the possible effect of the diurnal variation in the tilt angle of the geomagnetic dipole axis on the degree of illumination and the rapid change (for a few minutes) of the FAC intensity distribution sign have been obtained using selected events and observational data only from Northern Hemisphere stations. We are therefore planning follow-up studies with data from two hemispheres.

The work was financially supported by Basic Research program II.16 and in part by RFBR (Grant No. 18-0500437). The experimental data was obtained using the equipment of Shared Equipment Center "Angara" [http:/ ckp-angara.iszf.irk.ru]. We thank leaders of the international projects CARISMA, INTERMAGNET, GIMA, MACCS, and IMAGE, as well as magnetic networks in the Arctic and Antarctic (Arctic and Antarctic Research Institute and DMI) for 1-minute data from ground magnetometers, and researchers of some Russian and Japanese magnetic observatories for providing magnetic data used in this study. We took the available geomagnetic data directly from INTERMAGNET

[http://www.intermagnet.org/data-donnee/download-e ng.php], IMAGE [http://space.fmi./Image], MACCS [http://space.augsburg.edu/maccs/ requestdatafile.jsp], GIMA [https://www.gi.alaska.edu/monitors/ magnetometer], CARISMA [http://www.carisma.ca].

Key SW parameters and the $A E$ index were obtained through the SuperMAG website [http://supermag. jhuapl.edu].

\section{REFERENCES}

Atkinson G., Hutchison D. Effect of the day night ionospheric conductivity gradient on polar cap convective flow. $J$. Geophys. Res.: Space Phys. 1978, vol. 83, no. A2, pp. 725729. DOI: $10.1029 / \mathrm{JA0} 03 \mathrm{iA02p00725.}$

Benkevich L. Effects of ionospheric conductance in high latitude phenomena: PhD Thesis, University of Saskatchewan: Saskatoon, Canada, 2006, 210 p.
Benkevich L., Lyatsky W., Cogger L.L. Field-aligned currents between conjugate hemispheres. J. Geophys. Res.: Space Phys. 2000, vol. 105, no. A12, pp. 27727-27737. DOI: $10.1029 / 2000 j a 900095$.

Boroev P.H., Gelberg M.G. Dependence of the longitudinal localization of the substorm center in geosynchronous orbits on the IMF Vy component. Geomagnetism and Aeronomy. 2001, vol. 41, no. 5, pp.588-594. (In Russian).

Cowley S.W.H. Magnetospheric asymmetries associated with the y-component of the IMF. Planet. Space Sci. 1981, vol. 29, no. 1, pp. 79-96. DOI: 10.1016/0032-0633(81)90141-0.

Cowley S.W.H., Lockwood M. Excitation and decay of solar-wind driven flows in the magnetosphere-ionosphere system. Ann. Geophys. 1992, vol. 10, pp. 103-115.

Coxon J.C., Milan S.E., Anderson B.J. A Review of Birkeland Current Research Using AMPERE. Electric Currents in Geospace and Beyond. Eds. A. Keiling et al. Hoboken, New Jersey, USA. 2018, pp. 259-278. DOI: 10.1002/9781119 324522.ch16.

Forsyth C., Shortt M., Coxon J.C., et al. Seasonal and temporal variations of field-aligned currents and ground magnetic deflections during substorms. J. Geophys. Res.: Space Phys. 2018, vol. 123, no. 4, pp. 2696-2713. DOI: $10.1002 / 2017$ ja025136.

Haaland S., Lybekk B., Maes L., et al. North-south asymmetries in cold plasma density in the magnetotail lobes: Cluster observations. J. Geophys. Res.: Space Phys. 2017, vol. 122, no. 1 , pp. 136-149. DOI: 10.1002/2016ja023404.

Iijima T., Potemra T.A. Large-scale characteristics of field-aligned currents associated with substorms. J. Geophys. Res.: Space Phys. 1978, vol. 83, no. A2, pp. 599615. DOI: 10.1029/JA083iA02p00599.

Kostarev D. V., Mager P. N., Klimushkin D. Yu. Alfvén wave parallel electric field in the dipole model of the magnetosphere: Gyrokinetic treatment. J. Geophys. Res.: Space Phys. 2021, vol. 126, e2020JA028611. DOI: 10.1029/2020JA028611.

Liou K., Mitchell E.J. Hemispheric asymmetry of the premidnight aurora associated with the dawn-dusk component of the interplanetary magnetic field. J. Geophys. Res.: Space Phys. 2019, vol. 124, pp. 1625-1634. DOI: 10.1029/ 2018JA025953.

Lukianova R., Kozlovsky A. Dynamics of polar boundary of the auroral oval derived from the IMAGE satellite data. Cosmic Res. 2013, vol. 51, no. 1, pp. 46-53. DOI: 10.1134/ s0010952513010061.

Lunyushkin S.B., Mishin V.V., Karavaev Y.A., et al. Studying the dynamics of electric currents and polar caps in ionospheres of two hemispheres during the August 17, 2001 geomagnetic storm. Solar-Terr. Phys. 2019, vol. 5, no. 2, pp. 15-27. DOI: $10.12737 / \mathrm{stp}-52201903$.

Lyatskaya S., Khazanov G.V., Zesta E. Interhemispheric field-aligned currents: Simulation results. J. Geophys. Res.: Space Phys. 2014, vol. 119, no. 7, pp. 5600-5612. DOI: 10.1002/ $2013 \mathrm{ja} 019558$.

Lyatskaya S., Lyatsky W., Zesta E. Effect of interhemispheric currents on equivalent ionospheric currents in two hemispheres: Simulation results. J. Geophys. Res.: Space Phys. 2015, vol. 121, no. 2, pp. 1339-1348. DOI: 10.1002/ $2015 \mathrm{ja} 021167$.

Lyatsky W.B. Tokovye sistemy magnitosferno-ionosfernyh vozmushchenii [Current Systems of MagnetosphericIonospheric Disturbances]. Leningrad, Nauka Publ., 1978, 199 p. (In Russian).

Lyatsky W.B., Maltsev Yu.P. Magnitosferno-ionosfernoe vzaimodeistvie [The Magnetosphere-Ionosphere Interaction]. Moscow, USSR, Nauka Publ., 1983, 192 p. (In Russian).

Mishin V.M. Spokojnye geomagnitnye variatsii i toki v magnitosfere [Quiet geomagnetic variations and currents in the mag- 
netosphere]. Novosibirsk, Nauka, 1976, 203 p. (In Russian).

Mishin V.M. The magnetogram inversion technique and some applications. Space Sci. Rev. 1990, vol. 53, no. 1-2, pp. 83-163. DOI: 10.1007/bf00217429.

Mishin V.M., Kurikalova M.A. Magnetospheric substorms in the Earth two hemispheres. The 8 March 2008 and 6 April 2000 events. Danish Scientific J. 2020, vol. 2, no.42, pp. 7-21.

Mishin V.M., Bazarzhapov A.D., Saifudinova T.I., et al. Different Methods to Determine the Polar Cap Area. J. Geomagnetism and Geoelectricity. 1992, vol. 44, no. 12, pp. 1207-1214. DOI: 10.5636/jgg.44.1207.

Mishin V.M., Kurikalova M.A., Förster M. Electric circuits and their generators in the Earth's magnetosphere: The concept of electric circuits as applied to the first phase of the April 6, 2000 superstorm. Geomagnetism and Aeronomy. 2010, vol. 50, no. 8, pp. 978-987. DOI: 10.1134/s0016 793210080086

Mishin V.M., Förster M., Kurikalova M.A., Mishin V.V. The generator system of field-aligned currents during the April 06, 2000, superstorm. Adv. Space Res. 2011, vol. 48, no. 7, pp. 1172-1183. DOI: 10.1016/j.asr.2011.05.029.

Mishin V.M., Kurikalova M.A., Mishin V.V., et al. Fieldaligned current dynamics in two selected intervals of the 6 April 2000 superstorm. Proc. XXXVIII Annual Seminar "Physics of Auroral Phenomena". Apatity, Russia, 2015a, pp. 24-27.

Mishin V.M., Mishin V.V., Kurikalova M.A., et al. Fieldaligned current dynamics during two substorms of summer and winter types and model for the electric circuit of the magnetosphere-ionosphere system of two hemispheres. Proc. XXXVIII Annual Seminar "Physics of Auroral Phenomena". Apatity, Russia, 2015b, pp. 28-31.

Mishin V.M., Mishin V.V., Moiseev A.V. Distribution of the field-aligned currents in the ionosphere: dawn-dusk asymmetry and its relation to the asymmetry between the two hemispheres. Geomagnetism and Aeronomy. 2016, vol. 56, no. 5, pp. 524-534. DOI: 10.7868/S0016794016050096.

Mishin V.M., Mishin V.V, Kurikalova M.A., et al. Positive and negative feedbacks in the magnetosphere-ionosphere coupling. J. Atmos. Solar-Terr. Phys. 2019, vol. 187, pp. 1021. DOI: 10.1016/j.jastp.2019.03.002.

Moses J.J., Siscoe G.L., Crooker N.U., Gorney D.J. IMF $B_{y}$ and day-night conductivity effects in the expanding polar cap convection model. J. Geophys. Res.: Space Phys. 1987, vol 92, no. A2, pp. 1193-1198. DOI: 10.1029/JA092iA02p01193.

Pettigrew E.D., Shepherd S.G., Ruohoniemi J.M. Climatological patterns of high-latitude convection in the Northern and Southern hemispheres: Dipole tilt dependencies and interhemispheric comparisons. J. Geophys. Res.: Space Phys. 2010, vol. 115, no. A7, pp. A07305. DOI: 10.1029/2009ja014956.

Reistad J.P., Østgaard N., Laundal K.M., et al. Observations of asymmetries in ionospheric return flow during different levels of geomagnetic activity. J. Geophys. Res.: Space Phys. 2018, vol. 123, no. 6, pp. 4638-4651. DOI: 10.1029/ $2017 \mathrm{ja} 025051$.

Reistad J.P., Laundal K.M., Østgaard N., et al. Separation and quantification of ionospheric convection sources: 2 . The dipole tilt angle influence on reverse convection cells during northward IMF. J. Geophys. Res.: Space Phys. 2019, vol. 124, no. 7, pp. 6182-6194. DOI: 10.1029/2019ja026641.

Richmond A.D., Roble R.G. Electrodynamic effects of thermospheric winds from the NCAR Thermospheric General Circulation Model. J. Geophys. Res.: Space Phys. 1987, vol. 92, no. A11, pp. 12365-12376. DOI: 10.1029/JA092iA11p12365.

Senior C., Fontaine D., Caudal G., et al. Convection electric fields and electrostatic potential over $61^{\circ}<\Lambda<72^{\circ}$ invariant latitude observed with the European incoherent scatter facility: 2. Statistical results. Ann. Geophys. 1990, vol. 8, pp. 257-272.
Shue J.-H. Dependence of the ionospheric convection pattern on the conductivity and the southward IMF: Ph. D. Thesis. University of Alaska, Fairbanks, 1993, 141 p.

Shirapov D.S., Mishin V.M. Modelirovanie global'nykh elektrodinamicheskikh processov $v$ geomagnitosfere [Modeling of the Global Electrodynamic Processes in the Geomagnetosphere]. Ulan-Ude, East Siberian State Technological University Publ., 2009, 213 p. (In Russian).

Stenbaek-Nielsen H.C., Otto A. Conjugate auroras and the interplanetary magnetic field. J. Geophys. Res.: Space Phys. 1997, vol. 102, no. A2, pp. 2223-2232. DOI: 10.1029/96ja03563.

Suvorova A.V. Flux enhancements of $>30 \mathrm{keV}$ electrons at low drift shells $L<1.2$ during last solar cycles. $J$. Geophys. Res.: Space Phys. 2017, vol. 122, pp. 12,27412,287. DOI: $10.1002 / 2017 J A 024556$.

Tenfjord P., Østgaard N., Snekvik K., et al. How the IMF $B_{y}$ induces a $B_{y}$ component in the closed magnetosphere and how it leads to asymmetric currents and convection patterns in the two hemispheres. J. Geophys. Res.: Space Phys. 2015, vol. 120, no. 11, pp. 9368-9384. DOI: 10.1002/2015ja021579.

Velichko V.A., Boroyev R.N., Gelberg M.G. Azimuthal asymmetry of field-aligned currents flowing in and out of the ionosphere in a substorm current wedge. Geomagnetism and Aeronomy. 2002a, vol. 42, no. 5, pp. 619-623. (In Russian).

Velichko V.A., Boroyev R.N., Gelberg M.G., et al. Northsouth asymmetry of the substorm intensity depending on the IMF $B_{y}$-component. Earth, Planets and Space. 2002b, vol. 54, pp. $955-961$.

Østgaard N., Reistad J.P., Tenfjord P., et al. The asymmetric geospace as displayed during the geomagnetic storm on August 17, 2001. Ann. Geophys. 2018, vol. 36, pp. 15771596. DOI: 10.5194/angeo-2018-65.

URL: http://ckp-angara.iszf.irk.ru/index_en.html (accessed June 18, 2020).

URL: http://www.intermagnet.org/data-donnee/downloadeng.php (accessed June 18, 2020).

URL: http://space.fmi./Image (accessed June 18, 2020).

URL: http://space.augsburg.edu/maccs/requestdatafile.jsp (accessed June 18, 2020).

URL: https://www.gi.alaska.edu/monitors/magnetometer (accessed June 18, 2020).

URL: http://www.carisma.ca (accessed June 18, 2020).

URL: http://supermag.jhuapl.edu (accessed June 18, 2020).

How to cite this article

Mishin V.V., Mishin V.M., Kurikalova M.A. Dynamics of distribution asymmetry of field-aligned currents during substorms in the equinox season. Solar-Terrestrial Physics. 2021. Vol. 7. Iss. 1. P. 32 40. DOI: $10.12737 /$ stp-71202105 